\title{
A Review Paper on Detection of Optic Disc Damage using Retinal Images
}

\author{
Apeksha R. Padaria \\ M.E. (P.G. Student) \\ Computer Science and Engineering \\ Parul Institute of Technology
}

\author{
Bhailal Limbasiya \\ Assistant Professor \\ Computer Science and Engineering \\ Parul Institute of Technology
}

\begin{abstract}
Glaucoma is a chronic eye disease, which can be controlled but cannot be cured. If left untreated, loss of vision occurs gradually and even become blind. The detection and diagnosis of glaucoma is important. In glaucoma, Optic Cup (OC) is dependent on the type of glaucoma and the level of intra ocular pressure. Localization of Optic Disc (OD) simplifies the segmentation. OD and OC detection is a primary step in developing an automated algorithm for glaucoma detection. The paper provides overview of the different methods for optic cup and optic disc detection from retinal image.
\end{abstract}

\section{Keywords}

Glaucoma, Cup to Disc Ratio (CDR), ISNT, Fundus Image, Optic Disc Segmentation, Optic Cup Segmentation

\section{INTRODUCTION}

In today's time, there are lots of diseases that affect the normal life of a human. Lots of people in rural and semi-urban areas suffer from eye diseases such as Glaucoma, Diabetic Retinopathy, Age based Macular Degradation etc. Automatic retina image analysis is an important screening tool for early detection of risks and diseases. Glaucoma is a second leading cause of permanent blindness. It is known as "silent thief of sight". Glaucoma usually causes no symptoms and warnings; it can only be diagnosed by regular eye examination. It is caused by an increase in intraocular pressure within eye (Fig. 1). The optic nerve carries image information to brain. A liquid called "aqueous" is continuously flowing inside the eye. This liquid creates pressure on the internal surface of the eye. In normal eye this pressure is between 14 to $20 \mathrm{mmHg}$. If it is between 20 to $24 \mathrm{mmHg}$, it shows the symptoms of glaucoma. If the pressure is greater than $24 \mathrm{mmHg}$, it is detected as glaucoma. In healthy eyes, there is normal balance between the fluids, one that is produced in the eye, and the second that leaves the eye through eye's drainage system. This balance of fluids keeps Inter Ocular Pressure (IOP) constant in the eye. But in glaucoma, the balance of fluids produced in the eye is not maintained properly. As a result it causes an increase in IOP, resulting in the damage of optic nerve. Due to increase in IOP, the cup size begins to increase which consequently increases the Cup to Disc Ratio. As for normal disc the CDR is considered to be less than 0.5 but in case of glaucoma, it is greater than 0.5. As the cup size increases it also affects the Neuroretinal Rim (NRR).

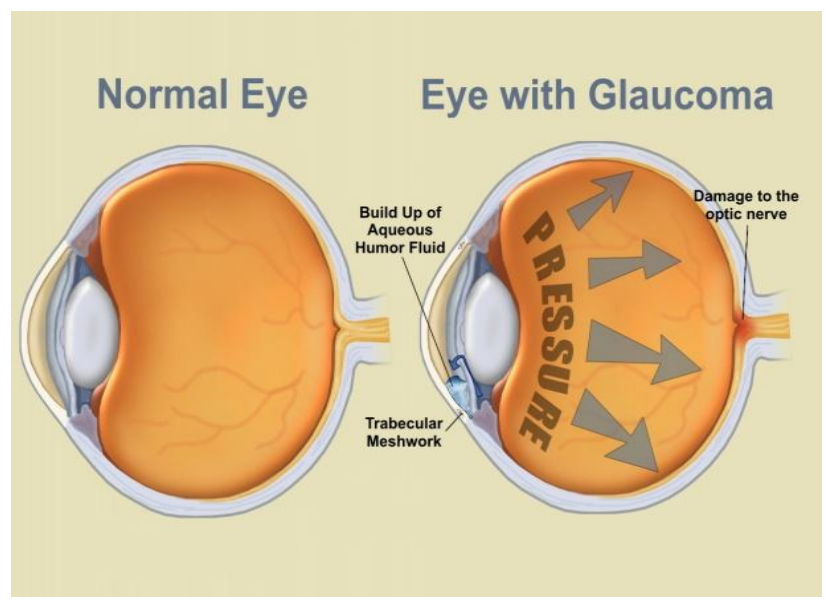

Fig 1: Comparison of Normal eye and eye with Glaucoma[9]

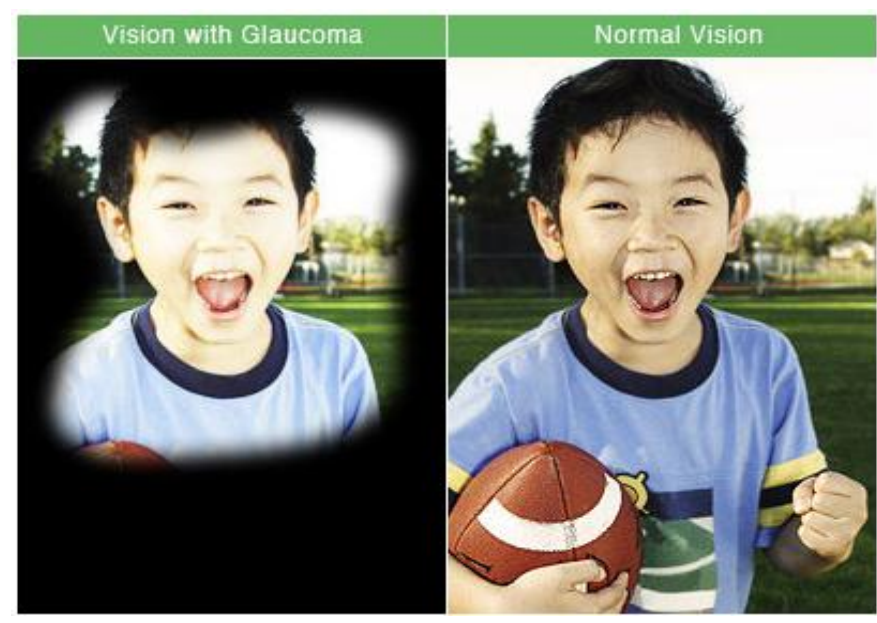

Fig. 2: Vision of Normal and Glaucoma [10]

The digital fundus image of a normal eye and glaucoma tic disc and inferior, superior, nasal and temporal (ISNT quadrants) are illustrated in Fig. 3.

The total blindness due to Glaucoma in the country today is 12.8 per cent. In India, approximately 90 per cent of Glaucoma cases go undiagnosed. Glaucoma affects approximately 12 million people in India and by 2020 it will become 16 million people. Statistics say that one person out of eight persons with age of 40 years or above in India is either suffering from Glaucoma or is at the risk of the disease [8]. 
With great improvement in field of medical imaging, Image processing technique helps in early diagnosis of glaucoma and other eye disease. Retinal fundus images assist trained clinicians to diagnose any abnormality and any change in retina. These images are captured by using special devices called ophthalmoscopes. Medical image analysis and processing has great significance in non-invasive treatment and clinical study. The information about the optic disc can be used to examine severity of glaucoma. The location of the optic disc is an important issue in retinal image analysis as it is a significant landmark feature. Fig. 4 shows the fundus camera and retinal fundus image.
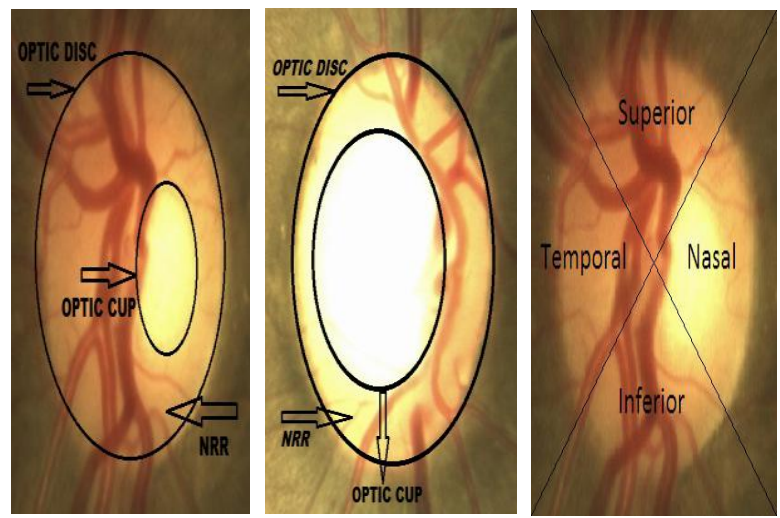

Fig. 3: L to R: Normal Disc $(C D R<0.5)$, Glaucoma tic Disc $($ CDR>0.5), ISNT Quadrants [1]
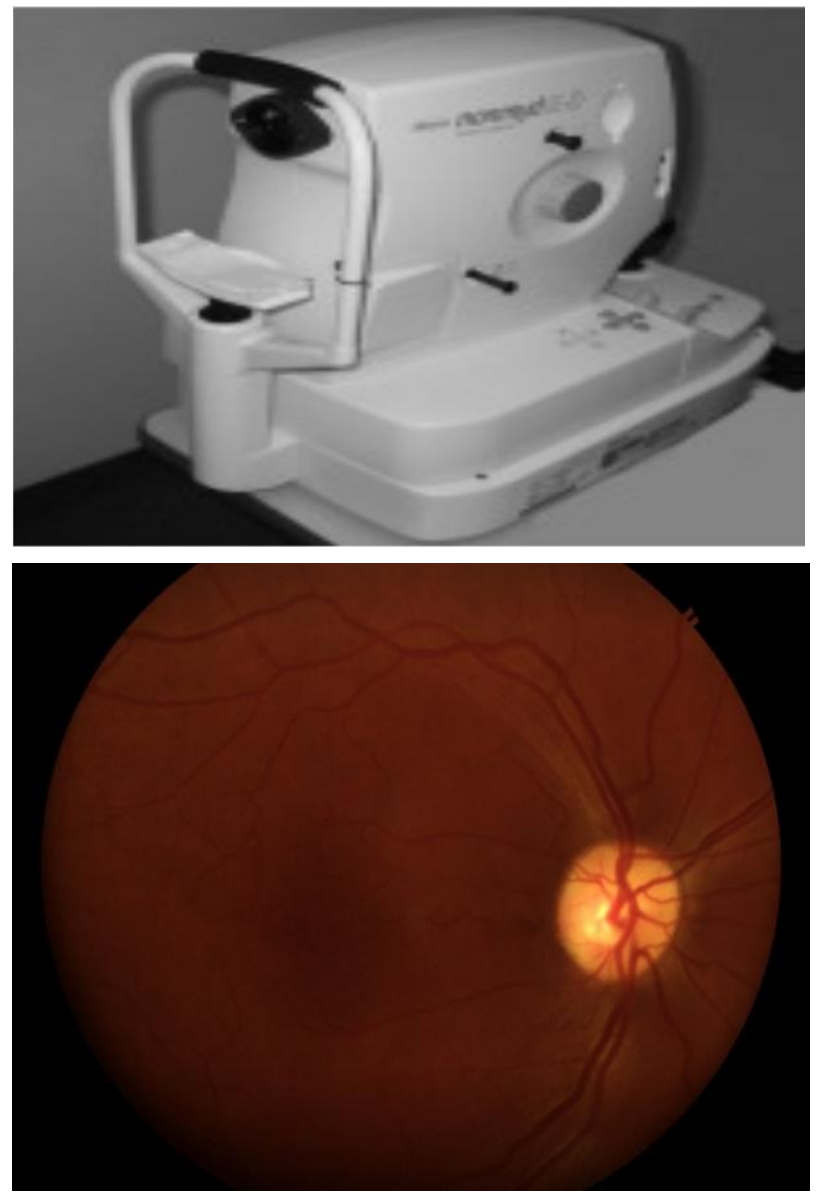

Fig. 4: Digital fundus camera and acquired retinal fundus image [2]
The paper is organized as follows: Section II depicts types of glaucoma, Section III describes different methods for detecting of optic cup and optic disc from retinal image and Section IV concludes the paper.

\section{TYPES OF GLAUCOMA}

The two main types are open-angle glaucoma and angleclosure glaucoma.

\subsection{Open-Angle Glaucoma}

It is the most common type of glaucoma which accounts for at least $90 \%$ of all other glaucoma causes.

- It is caused by the slow hindering of the drainage canals which is resulting in increased eye pressure.

- It has a wide open angle between the iris and cornea.

- It builds up slowly and is a long life condition.

- It has symptoms and damages are not noticed.

It is also named as "primary or chronic glaucoma". Openangle is the angle where the iris meets the cornea as wide and open as it should be.

\subsection{Angle-Closure Glaucoma}

It is a less common form of glaucoma.

- It is caused by blocked drainage canals and subsequently results in a sudden rise in intraocular pressure.

- It has a closed or narrow angle between the iris and cornea.

- It develops very quickly.

- It has symptoms and damage that are noticeable.

- It demands immediate medical attention.

It is also known as "acute glaucoma or narrow angle glaucoma". It is a result of the angle among the iris and cornea closing.

\subsection{Normal Tension Glaucoma}

- It is also called as low-tension or normal-pressure glaucoma.

- In this type of glaucoma damage occurs to the optic nerve without eye pressure which exceeds the normal range $(10-20 \mathrm{mmHg})$.

\subsection{Congenital Glaucoma}

- This type of glaucoma is noticed in babies in incorrect or incomplete development of the eye's drainage canals during the parental period.

- This is a rare condition which may be inherited.

- It is also referred as childhood glaucoma, paediatric or infantile glaucoma.

- It is normally diagnosed within the first year of baby life.

There are other types of glaucoma like Secondary Glaucoma, Pigmentary Glaucoma, Pseudoexfoliative Glaucoma, Traumatic Glaucoma, Neovascular Glaucoma, etc. 


\section{DIFFERENT METHODS FOR DETECTING OF OPTIC CUP AND OPTIC DISC FROM RETINAL IMAGE}

In 2014, Hafsah Ahmad performed a work, "Detection of Glaucoma using Retinal Fundus Images" [3]. This paper presented an image processing technique for the detection of glaucoma which mainly affects the optic disc due to increased cup size. Here Glaucoma is divided into two features using retinal fundus images, (i) Cup to Disc Ratio (CDR) (ii) Ratio of Neuroretinal Rim in ISNT quadrants. The novel method Morphological technique is used to extract above two features. The following methodology used in this paper:

\section{Preprocessing of an image \\ 2. Extraction of Optic Disc and Cup \\ 3. Extraction of Neuroretinal Rim \\ 4. Classification}

1. Preprocessing of an image: The main objective of preprocessing techniques is to remove image variation by normalizing the original retinal image without losing essential information. In coloured retinal fundus images, Optic disc appears to be the brightest part with light orange or pink color and is deemed to be Region of Interest. The green plane is extracted from original image for extraction of optic cup. The original image was then converted to HSV plane.

2. Extraction of Optic Disc and Cup: In this method original image green plane is extracted for extraction of optic cup and then converted to gray scale image. The gray scale image is then converted to binary image. Morphological operations such as dilation and erosion are used to fill the gaps due to presence of blood vessels. Gaussian filter is used for smoothing the boundaries of images. Edges of both optic disc and optic cup in resultant image are found by applying canny filter.

$$
\mathrm{CDR}=(\text { Cup area } / \text { Disc area }) * 2
$$

3. Extraction of Neuroretinal Rim: In this method the optic disc and optic cup are already extracted now in order to extract NRR AND operation is applied on both resultant images of cup and disc. On extracted NRR image a mask of size $256 \times 256$ is applied to measure the ratio of area covered by neuroretinal rim in ISNT quadrants.

4. Classification: Classification of glaucoma has been done using the above mentioned two extracted features.

Usually misclassification rate is described by the correct and false positive and correct and false negative parameters as follows:

$$
\text { Accuracy }=\left(\frac{\mathrm{Cp}+\mathrm{Cn}}{\mathrm{Cp}+\mathrm{Cn}+\mathrm{Fp}+\mathrm{Fn}}\right) * 100
$$

Here $C p$ represents True Positive, $C n$ represents True Negative, $F p$ represents False Positive and Fn represents False Negative.

In April 2013, Mei Hui Tan performed a work, "Automatic Notch Detection In Retinal Images"[4]. The proposed algorithm consists of four main steps:
1. Disc and vessel segmentation

2. Vessel bend computation

3. Feature points selection

4. Classification of the images using $\mathrm{I}$ and $\mathrm{T}$ values.

The proposed method is able to determine the presence of notching in the optic cup from color fundus images.

Preliminary results show that the algorithm is automatic, efficient and accurate

In September 2013, DharmannaLamani performed a work," Cup-Disk Segmentation and Fractal Dimension to Detect Glaucomatous Eyes" [5]. It detects glaucoma through cupdisc segmentation \& fractal dimension using image analysis NRR. This paper uses:

(1) Semi variance method is more efficient.

(2) Fractal dimension feature can be used for earlier detection of glaucoma.

(3) The visualization of segmentation exposes decrease in area of signifies attacking glaucoma neuroretinal rim.

(4) Fractal Dimension (FD) is noticed in the range of 1.50 to 1.59 .

In September 2011, Fengshou Yin performed a work,"Model-based Optic Nerve Head Segmentation on Retinal Fundus Images" [6].The following method used in this paper:

1. Shape and Appearance Modeling

2. Pre-processing

3. Edge Detection and Circular Hough Transform

4. Optic Disc Boundary Extraction

In May 2014, Sushma G. Thorat performed a work, "Automated Glaucoma Screening using CDR from 2D Fundus Images" [7]. This paper focuses on automated glaucoma screening using CDR from 2D fundus Images. Aim of this paper is classification based disc and cup segmentations for glaucoma screening. In this proposed approach, preprocessing like color contrast enhancement, image filtration are performed. It is accepted by a combined approach for image segmentation and classification, thresholding and morphological operation. K-Means clustering, Gabor wavelet transformations are also used to receive exact boundary delineation. Prior knowledge of the cup is incorporated by including location information for cup segmentation. CDR is computed for glaucoma screening based on the segmented disc and cup.

\section{CONCLUSION}

From the review of the above papers, it can be concluded that many different techniques can be used for detection of optic cup and optic disc from retinal image. Glaucoma is the second main cause of blindness in the world. Hence its detection and diagnosis are very essential. Different manual and automatic glaucoma detection methods are available. Different authors are noted that there is a constant research happening in this field. Here an attempt is done to learn and understand some of the techniques used till now for detection and extraction of the features. 


\section{REFERENCES}

[1] Fauzia Khan, Shoaib A. Khan, Ubaid Ullah Yasin, Ihtisham ul Haq, Usman Qamar,"Detection of Glaucoma Using Retinal Fundus Images" The 2013 Biomedical Engineering International Conference 2013

[2] Preeti, Jyotika Pruthi," Review of Image Processing Technique for Glaucoma Detection" IJCSMC, Vol. 2, Issue. 11, November 2013, pg.99 - 105

[3] Hafsah Ahmad, Aqsa Shakeel, Syed Omer Gillani, Umer Ansari, "Detection of Glaucoma Using Retinal Fundus Images" April 2014 IEEE

[4] Mei Hui Tan, Ying Sun, Sim Heng Ong, Jiang Liu, Mani Baskaran, Tin Aung, Tien Yin Wong, "Automatic Notch Detection In Retinal Images" 2013 IEEE 10th International Symposium on Biomedical Imaging From Nano to Macro San Francisco, CA, USA, April 7-11, 2013
[5] DharmannaLamani1, T. C. Manjunath2, Ramegowda3,"Cup-disk Segmentation and Fractal Dimension to Detect Glaucomatous Eyes" Research \& Technology in the CRT 2013, National Conference on Challenges in September 2013.

[6] Fengshou Yin, Jiang Liu, Sim Heng Ong, Ying Sun, Damon, "Model-based Optic Nerve Head Segmentation on Retinal Fundus Images" 33rd Annual International Conference of the IEEE EMBS Boston, Massachusetts USA, September 2011

[7] Sushma G.Thorat M.E.(Electronics and Telecommunication), “ Automated Glaucoma Screening using CDR from 2D Fundus Images" International Journal of Computational Engineering Research (IJCER) ISSN (e): 2250 - 3005 || Vol, 04 || Issue, 5 || May - 2014 